

А. Н. Васильев, М. М. Перекалин, Флуктуационно-диссипационная теорема для составных операторов в моделях критической динамики, ТМФ, 1999, том 118, номер 2, 305-310

DOI: https://doi.org/10.4213/tmf701

Использование Общероссийского математического портала Math-Net.Ru подразумевает, что вы прочитали и согласны с пользовательским соглашением

http: //www . mathnet.ru/rus/agreement

Параметры загрузки:

IP : 54.89 .56 .158

26 апреля 2023 г., 16:41:51 


\section{ФЛУКТУАЦИОННО-ДИССИПАЦИОННАЯ ТЕОРЕМА ДЛЯ СОСТАВНЫХ ОПЕРАТОРОВ В МОДЕЛЯХ КРИТИЧЕСКОЙ ДИНАМИКИ}

Приводятся общая формулировка и доказательство флуктуационно-диссипационной теоремы для составных операторов в произвольной модели критической динамики.

\section{1. ОБЩЕЕ ОПИСАНИЕ МОДЕЛЕЙ КРИТИЧЕСКОЙ ДИНАМИКИ}

Любая конкретная модель критической динамики для поля или (в обшем случае) семейства полей $\varphi \equiv\left\{\varphi_{a}(x), x \equiv t, \mathbf{x}\right\}$ задается статическим функционалом действия $S^{\mathrm{st}}(\varphi)$ и системой стохастических уравнений

$$
\begin{gathered}
\partial_{t} \varphi_{a}(x)=\left(\alpha_{a b}+\beta_{a b}\right) H_{b}(x ; \varphi)+\eta_{a}(x), \\
\left\langle\hat{\eta}_{a}(x) \hat{\eta}_{b}\left(x^{\prime}\right)\right\rangle=2 \alpha_{a b} \delta\left(x-x^{\prime}\right)
\end{gathered}
$$

(по повторяющимся индексам типа $b$ в (1a) предполагается суммирование), в которых

$$
\left.H_{b}(x ; \varphi) \equiv \frac{\delta S^{\mathrm{st}}(\varphi)}{\delta \varphi_{b}(\mathbf{x})}\right|_{\varphi(\mathbf{x}) \rightarrow \varphi(x)}
$$

$\alpha_{a b}$ - симметричная и положительно-определенная матрица коэффициентов Онсагера, $\beta_{a b}$ - антисимметричная матрица коэффициентов межмодовых связей, $\hat{\eta}_{a}$ - набор случайных источников ("шумов") с гауссовым распределением, заданным коррелятором (1б) и условием $\left\langle\hat{\eta}_{a}\right\rangle=0, \eta_{a}$ в $(1 \mathrm{a})$ - любая конкретная реализация случайной величины $\hat{\eta}_{a}$. В дальнейшем всегда будет иметься в виду “стандартная постановка" задачи (1): она рассматривается на всей оси времени $-\infty<t<+\infty$ с дополнительным условием запаздывания и нулевыми асимптотическими условиями для полей при $|x| \rightarrow \infty$.

Коэффициенты $\alpha_{a b}$ в (1) не зависят от полей $\varphi$ и в конкретных моделях являются либо простыми константами, либо (при наличии "сохранения" для соответствующих компонент $\varphi_{a}$ ) величинами типа const $\cdot \partial^{2}$, где $\partial^{2}$ - оператор Лапласа. В отличие от

\footnotetext{
* Санкт-Петербургский государственный университет, Санкт-Петербург, Россия
} 
$\alpha$ в коэффициентах межмодовой связи $\beta_{a b}$ допускается локальная зависимость от полей $\varphi$ и от их пространственных (но не временных) производных $\partial \varphi$. В трансляционно-инвариантных моделях (которыми мы и будем ограничиваться) явная зависимость от переменных $x=t, \mathbf{x}$ в коэффициентах $\alpha$ и $\beta$ не допускается. Для правильного согласования динамики и статики (см. ниже) коэффициенты $\beta$ в (1) должны удовлетворять соотношениям

$$
\beta_{a b}=-\beta_{a b}^{\mathrm{T}}, \quad \frac{\delta \beta_{a b}(x ; \varphi)}{\delta \varphi_{a}(x)}=0,
$$

первое из которых означает антисимметричность матрицы $\beta$ (символ транспонирования Т относится к возможным операциям типа $\partial$ в коэффициентах $\beta_{a b}, \partial^{\mathrm{T}}=-\partial$ ).

Уравнение Фоккера-Планка для функции распределения $P_{t}(\varphi)$ полей $\varphi(t ; \mathbf{x})$ в один момент времени $t$ записывается для системы (1) следующим образом:

$$
\left\{\partial_{t}+\frac{\delta}{\delta \varphi_{a}}\left[\left(\alpha_{a b}+\beta_{a b}\right) \frac{\delta S^{\mathrm{st}}}{\delta \varphi_{b}}-\alpha_{a b} \frac{\delta}{\delta \varphi_{b}}\right]\right\} P_{t}(\varphi)=0
$$

(выражение в квадратных скобках и операция $\delta / \delta \varphi_{a}$ имеют аргумент $\mathbf{x}$, по которому подразумевается интегрирование), а его стационарным решением является функционал

$$
P(\varphi)=\text { const } \cdot \exp S^{\text {st }}(\varphi)
$$

т.е. обычное равновесное распределение для исходной статической модели с действием $S^{\text {st }}(\varphi)$, в этом и состоит общий принцип взаимной согласованности динамики и статики. При подстановке функционала (5) в уравнение (4) все вклады с коэффициентами $\beta$ исчезают в силу соотношений (3), т.е. межмодовая связь не влияет на одновременную (и совпадающую с равновесной при стандартной постановке задачи) функцию распределения.

Помимо основных соотношений (3), обеспечивающих отсутствие влияния на статику межмодового взаимодействия, последнее в конкретных моделях всегда удовлетворяет условию $t$-обратимости. Это значит, что динамическое уравнение (1a) с чисто межмодовой правой частью (т.е. без учета шумов и диссипативных вкладов с коэффициентами $\alpha$ ) инвариантно относительно операции отражения времени. Последней соответствует некоторое преобразование полей:

$$
\varphi(x) \rightarrow \varphi_{\mathrm{T}}(x)=I \varphi(\mathrm{T} x), \quad I^{2}=1, \quad \mathrm{~T} x \equiv \mathrm{T}\{t, \mathbf{x}\}=\{-t, \mathbf{x}\} .
$$

Задающая представление дискретной операции Т-отражения матрица $I=I^{-1}$ обычно (но не всегда) просто диагональна, и тогда ее диагональные элементы $\epsilon_{a}= \pm 1$ определяют $t$-четность соответствуюшей полевой компоненты $\varphi_{a}$. При Т-отражении (6) все времена и операция $\partial_{t}$ в динамическом уравнении (1a) меняют знак, в конкретных моделях функционал $S^{\text {st }}(\varphi)$ на полях $\varphi(x)$ всегда инвариантен относительно замены $\varphi(\mathbf{x}) \rightarrow I \varphi(\mathbf{x})$, поэтому его вариационная производная в (2) преобразуется с помощью транспонированной матрицы $I^{\mathrm{T}}=\left(I^{-1}\right)^{\mathrm{T}}$. Т-инвариантность (обратимость) межмодового взаимодействия означает, что вклад $\beta H$ в уравнении (1a) преобразуется точно 
так же, как производная $\partial_{t} \varphi$ в левой части, т.е. матрицей $-I$, тогда как диссипативный вклад $\alpha H$ преобразуется матрицей $I$, поэтому меняет знак относительно $\partial_{t} \varphi$. Все это обеспечивается следующими свойствами матриц $\alpha$ и $\beta=\beta(\mathbf{x} ; \varphi)$ с полем $\varphi(\mathbf{x})$ (статическая версия записи коэффициентов $\beta$ ) в уравнении (1a):

$$
|\alpha|^{\mathrm{T}}=\alpha, \quad|\beta(\mathbf{x} ; I \varphi)|^{\mathrm{T}}=-\beta(\mathbf{x} ; \varphi) .
$$

В конкретных динамических моделях А-J [1] соотношения (7) всегда выполняются.

Хорошо известно (см., например, $[2,3])$, что любая стохастическая задача типа (1) полностью эквивалентна квантово-полевой модели с удвоенным числом полей $\phi=\varphi, \varphi^{\prime}$ и функционалом действия

$$
S(\varphi)=\varphi^{\prime} \alpha \varphi^{\prime}+\varphi\left[-\partial_{t} \varphi+(\alpha+\beta) H(\varphi)\right]
$$

с $H(\varphi)$ из (2) и с подразумеваемыми нужными интегрированиями по $x$ и суммированиями по индексам. Эквивалентность означает, в частности, что динамические корреляционные функции $\langle\hat{\varphi} \ldots \hat{\varphi}\rangle$ случайных полей $\hat{\varphi}(x)$ совпадают с функциональными средними $\langle\varphi \ldots \varphi\rangle$ с весом $\exp S(\phi)$. То же справедливо для функций Грина всех полей $\phi=\varphi, \varphi^{\prime}$ и любых построенных из них составных операторов $F(\phi)$. В дальнейшем будем полагать

$$
\langle F(\phi)\rangle=\int D \phi F(\phi) \exp S(\phi),
$$

считая нормировочньй множитель включенным в меру $D \phi$.

\section{2. ФЛУКТУАЦИОННО-ДИССИПАЦИОННАЯ ТЕОРЕМА}

Динамические функции отклика на нестационарное внешнее поле $h=\left\{h_{a}(x)\right\}$ определяются как кратные вариационные производные по $h$ различных величин $\langle\hat{\varphi} \ldots \hat{\varphi}\rangle$ при $h=0$. Внешнему полю соответствует добавка

$$
h \varphi=\int d \mathbf{x} h(x) \varphi(\mathbf{x})
$$

к функционалу $S^{\mathrm{st}}(\varphi)$, т.е. аддитивная добавка $h_{b}(x)$ к $H_{b}(x ; \varphi)$ в уравнении $(1 \mathrm{a})$ и в динамическом действии (8). Поэтому простейшей из таких функций отклика является величина

$$
R_{a b}\left(x, x^{\prime}\right)=\left.\frac{\delta\left\langle\hat{\varphi}_{a}(x)\right\rangle}{\delta h_{b}\left(x^{\prime}\right)}\right|_{h=0}=\left\langle\varphi_{a}(x) \bar{\varphi}_{b}\left(x^{\prime}\right)\right\rangle
$$

здесь и далее символ $\langle\ldots\rangle$ обозначает функциональное усреднение по полям $\phi=\varphi, \varphi^{\prime} \mathrm{c}$ весом $\exp S(\phi)$ и

$$
\bar{\varphi} \equiv \varphi^{\prime}(\alpha+\beta)=(\alpha-\beta) \varphi^{\prime}
$$

(напомним, что матрица $\alpha$ симметрична, а $\beta$ антисимметрична и что $x M=M^{\mathrm{T}} x$ для любого вектора $x$ и произвольной матришы $M)$. 
Все функции отклика удовлетворяют естественным требованиям запаздывания, в частности для функции (9) $R_{a b}\left(x, x^{\prime}\right)=0$ при $t<t^{\prime}$ в аргументах $x=t, \mathbf{x}$ и $x^{\prime}=t^{\prime}, \mathbf{x}^{\prime}$.

Хорошо известная флуктуационно-диссипационная $(\Phi Д)$ теорема связывает функцию отклика (9) с динамическим коррелятором $\left\langle\varphi_{a}(x) \varphi_{b}\left(x^{\prime}\right)\right\rangle \equiv C_{a b}\left(x, x^{\prime}\right)$ :

$$
\left\langle\varphi_{a}(x) \bar{\varphi}_{b}\left(x^{\prime}\right)\right\rangle=\Theta\left(t-t^{\prime}\right)\left\langle\varphi_{a}(x) \partial_{t^{\prime}} \varphi_{b}\left(x^{\prime}\right)\right\rangle
$$

откуда с учетом свойства запаздьвания функции отклика и транслящионной инвариантности по времени всех величин следует

$$
R_{a b}(\omega, k)-R_{b a}(-\omega,-k)=i \omega C_{a b}(\omega, k)
$$

в импульсно-частотном представлении.

Мы хотим доказать справедливость следуюшего обобщения ФД-теоремы (11) для произвольной модели типа (1):

$$
\left\langle M(x) \cdot \bar{\varphi}\left(x^{\prime}\right) \cdot N\left(x^{\prime}\right)\right\rangle=\Theta\left(t-t^{\prime}\right)\left\langle M(x) \cdot \partial_{t^{\prime}} \varphi\left(x^{\prime}\right) \cdot N\left(x^{\prime}\right)\right\rangle
$$

где $M$ и $N$ - любые построенные только из полей $\varphi\left(\right.$ без $\left.\varphi^{\prime}\right)$ и их производных локальные (в общем случае многокомпонентные) составные операторы, преобразующиеся линейно при Т-отражении полей (6). Последнее означает, что при замене $\varphi(x) \rightarrow \varphi_{\mathrm{T}}(x)$ в $M(x)$ получается новый объект $M^{\prime}=M_{I}(\mathrm{~T} x)$, все компоненты которого являются некоторыми линейными комбинациями компонент исходного оператора $M$ с точностью до замены $x \rightarrow$ Т $x$ аргументов входящих в $M$ полей $\varphi$ и символов $\partial$, и аналогично для $N(x)$ (индекс типа $b$ в (11) у полей $\bar{\varphi}$ и $\varphi$ в (13) подразумевается).

Переходя к доказательству ФД-теоремы (13), прежде всего заметим, что функцию отклика в левой части (13) можно представить в виде

$$
\begin{aligned}
R\left(x, x^{\prime}\right) & \equiv\left\langle M(x) \cdot \bar{\varphi}\left(x^{\prime}\right) \cdot N\left(x^{\prime}\right)\right\rangle= \\
& =\left.\frac{\delta}{\delta a\left(x^{\prime}\right)} \int D \phi M(x) \exp \left[S(\phi)+\varphi^{\prime}(\alpha+\beta) N a\right]\right|_{a=0}
\end{aligned}
$$

где $a \equiv\{a(x)\}$ - дополнительные "источники" с нужными для обеспечения скалярности вклада $\varphi^{\prime}(\alpha+\beta) N a=\bar{\varphi} N a$ в (14) индексами (примеры: $\bar{\varphi}_{b} N a_{b}, \bar{\varphi}_{b} N_{b} a, \bar{\varphi}_{b} N_{c} a_{b c}$, $\bar{\varphi}_{b} N_{b c} a_{c}$ и т.п.).

Если подставить в (14) выражение (8) для динамического действия и выполнить сначала гауссово функциональное интегрирование по $\varphi^{\prime}$ (напомним, что величины $M$ и $N$ зависят по условию только от полей $\varphi$, а формальная “расходимость" интеграла по $\varphi^{\prime}$ для действия (8) с $\alpha>0$ значения не имеет и может быть при желании устранена заменой $\left.\varphi^{\prime} \rightarrow i \varphi^{\prime}\right)$ и лишш после этого вариационное дифференцирование по источнику $a$, то для функции отклика (14) получим следующее представление:

$$
\begin{aligned}
-2 R\left(x, x^{\prime}\right) & =-2\left\langle M(x) \cdot \bar{\varphi}\left(x^{\prime}\right) \cdot N\left(x^{\prime}\right)\right\rangle= \\
& =C \int D \varphi M(x) A\left(x^{\prime}\right) N\left(x^{\prime}\right) \exp \widetilde{S}(\varphi) \equiv\left\langle\left\langle M(x) A\left(x^{\prime}\right) N\left(x^{\prime}\right)\right\rangle\right\rangle,
\end{aligned}
$$


где $C$ - несушественная константа, $\langle\langle\ldots\rangle\rangle$ - удобное для дальнейшего обозначение функционального интегрирования по полям $\varphi$ с весом $C \exp (\widetilde{S}(\varphi))$ и

$$
\begin{aligned}
& \widetilde{S}=-\frac{1}{4}\left(F \alpha^{-1} F\right), \quad F \equiv-\partial_{t} \varphi+(\alpha+\beta) H, \\
& A=F \alpha^{-1}(\alpha+\beta)=\left(1-\beta \alpha^{-1}\right) F \equiv-\partial_{t} \varphi+B, \\
& B \equiv \alpha H+\beta \alpha^{-1}\left(\partial_{t} \varphi-\beta H\right)
\end{aligned}
$$

с функционалом $H$ из (2). Величины $\varphi, F, A, B$ нужно понимать как векторы по индексам полей $\varphi, \varphi^{\prime}$, величины $\alpha, \alpha^{-1}, \beta$ - как действуюшие на них справа или слева матрицы (перенос матрицы слева направо или наоборот сопровождается ее транспонированием, $\left.\alpha^{\mathrm{T}}=\alpha, \beta^{\mathrm{T}}=-\beta\right), \widetilde{S}(\varphi)$ в $(16 \mathrm{a})-$ квадратичная форма с ядром $\alpha^{-1}$, нужные аргументы $x$ и интегрирования по ним везде подразумеваются.

Как было показано (раздел 1 ), при Т-отражении (6) величины $\partial_{t} \varphi$ и $\beta H$ преобразуются матрицей $-I$, а $\varphi$ и $\alpha H$ - матрицей $I$. Величины первого типа будем называть "I-нечетными", а второго - "I-четными". C помощью соотношений (7) нетрудно убедиться, что все вклады в выражении (16в) для $B$ являются $I$-четными (отметим, что при подстановке $F$ из (16а) в выражение (16б) происходит взаимное сокрашение двух $I$-нечетных вкладов). В обозначениях (15), (16) имеем

$$
-2 R\left(x ; x^{\prime}\right)=\left\langle\left\langle M(x)\left[-\partial_{t^{\prime}} \varphi\left(x^{\prime}\right)+B\left(x^{\prime}\right)\right] \cdot N\left(x^{\prime}\right)\right\rangle\right\rangle,
$$

причем величины $\partial_{t^{\prime}} \varphi\left(x^{\prime}\right)$ и $B\left(x^{\prime}\right)$ имеют разную $I$-четность.

Покажем теперь, что действие $\widetilde{S}(\varphi)$ в $(15)$ Т-четно, т.е. инвариантно относительно Т-отражения (6). В квадратичной форме $F \alpha^{-1} F$ выражения (16а) для $\widetilde{S}$ "вектор" $F$ есть сумма $I$-четного и $I$-нечетного вкладов. Из первого равенства (7) следует, что в форме $F \alpha^{-1} F$ произведения вкладов $F$ с одинаковой $I$-четностью дают Т-четный вклад в $\widetilde{S}$. Поэтому Т-нечетный вклад может порождаться только перекрестным членом формы $F \alpha^{-1} F$, кратным выражению

$$
\left[-\partial_{t} \varphi+\beta H\right] \alpha^{-1}[\alpha H]=H\left[-\partial_{t} \varphi+\beta H\right] .
$$

Оба вклада в правой части равны нулю: второй вследствие антисимметричности матрицы $\beta$, а первый сводится к интегралу от полной производной. Действительно, в подробной записи (см.(2)) этот вклад имеет вид

$$
-\left.\int d x \partial_{t} \varphi(x) \frac{\delta S^{\mathrm{st}}(\varphi)}{\delta \varphi(\mathbf{x})}\right|_{*}=-\int d t \partial_{t} L(t),
$$

где

$$
\left.L(t) \equiv S^{\mathrm{st}}(\varphi)\right|_{*},
$$

а знак $\left.\right|_{*}$ везде обозначает замену $\varphi(\mathbf{x}) \rightarrow \varphi(x)$. 
Таким образом, действие $\widetilde{S}(\varphi)$ Т-инвариантно, поэтому инвариантны и все средние $\langle\langle\ldots\rangle\rangle$ с весом $\exp \widetilde{S}(\varphi)$. В частности, с учетом $I$-четности $B$ и $I$-нечетности $\partial_{t} \varphi$ имеем

$$
\left\langle\left\langle M(x) \cdot\left[-\partial_{t^{\prime}} \varphi\left(x^{\prime}\right)+B\left(x^{\prime}\right)\right] \cdot N\left(x^{\prime}\right)\right\rangle\right\rangle=\left\langle\left\langle M_{I}(\bar{x}) \cdot I\left[\partial_{\bar{t}^{\prime}} \varphi\left(\bar{x}^{\prime}\right)+B\left(\bar{x}^{\prime}\right)\right] \cdot N_{I}\left(\bar{x}^{\prime}\right)\right\rangle\right\rangle,
$$

где $\bar{x} \equiv \mathrm{T} x, \bar{x}^{\prime} \equiv \mathrm{T} x^{\prime}$ (см. (6)), а $M_{I}$ и $N_{I}$ - Т-преобразованные функционалы $M$ и $N$ (см. текст после соотношения (13)).

Учтем теперь свойство запаздывания функций отклика, из которого следует, что правая часть равенства $(17)$ исчезает при $t<t^{\prime}$, т.е.

$$
\left\langle\left\langle M(x) \cdot \partial_{t^{\prime}} \varphi\left(x^{\prime}\right) \cdot N\left(x^{\prime}\right)\right\rangle\right\rangle=\left\langle\left\langle M(x) \cdot B\left(x^{\prime}\right) \cdot N\left(x^{\prime}\right)\right\rangle\right\rangle \text { при } t<t^{\prime} .
$$

Возьмем в (19) $t>t^{\prime}$, тогда в правой части этого равенства будет $\bar{t}<\bar{t}^{\prime}$ и для нее можно воспользоваться соотношением (20) (здесь важно, что все компоненты $M_{I}$ и $N_{I}$ являются по условию некоторыми линейными комбинациями компонент исходных величин $M$ и $N$ с точностью до замены аргументов). Оно позволяет при $t>t^{\prime}$ заменить всю правую часть равенства (19) удвоенным вкладом ее первого слагаемого, совпадаюшим с вкладом первого слагаемого в левой части (19) вследствие Т-инвариантности. Таким образом, при $t>t^{\prime}$ имеем

$$
\left\langle\left\langle M(x) \cdot\left[-\partial_{t^{\prime}} \varphi\left(x^{\prime}\right)+B\left(x^{\prime}\right)\right] \cdot N\left(x^{\prime}\right)\right\rangle\right\rangle=-2\left\langle\left\langle M(x) \cdot \partial_{t^{\prime}} \varphi\left(x^{\prime}\right) \cdot N\left(x^{\prime}\right)\right\rangle\right\rangle,
$$

откуда с учетом соотношений (15), (17) и обшего свойства запаздывания функций отклика получаем равенство (13), т.е. обобшение $\Phi$ Д-теоремы для составных операторов. Простая $\Phi$ Д-теорема (11) - частный случай обшего соотношения (13) с $M=\varphi$ и $N=1$.

Благодарности. Авторы благодарят за поддержку Российский фонд фундаментальных исследований (грант 96-02-17033) и Конкурсный центр фундаментального естествознания Минобразования РФ (грант 97-0-14.1-30).

\section{Список литературы}

[1] P. C. Honenberg, B. I. Halperin. Rev. Mod. Phys. 1977. V. 49. P. 435.

[2] C. De Dominicis, L. Peliti. Phys. Rev. B. 1978. V. 18. P. 353.

[3] Л. Ц. Аджемян, А. Н. Васильев, Ю. М. Письмак. ТМФ. 1983. Т. 57. С. 268.

Поступила в редакцию 25.V.1998 г. 